\title{
Correlation Between the Clinical Severity, Bacterial Load, and Inflammatory Reaction in Children with Mycoplasma Pneumoniae Pneumonia*
}

\author{
Chen ZHANG ${ }^{\dagger}$, Qiao ZHANG ${ }^{\dagger}$, Jie-lin DU, Dan DENG, Ye-lei GAO, Cheng-lin WANG, Hong-jie ZHAO, Qian GUO, \\ Zhou FU, Dai-yin TIAN" \\ Department of Pulmonology, Children's Hospital of Chongqing Medical University, National Clinical Research Center for \\ Child Health and Disorders, Ministry of Education Key Laboratory of Child Development and Disorders, Chongqing 400014, \\ China
}

(C) Huazhong University of Science and Technology 2020

\begin{abstract}
Summary: Given the lack of defining features in the clinical manifestations and radiographic findings for children with mycoplasma pneumoniae pneumonia (MPP), quantitative polymerase chain reaction (qPCR) has become a useful diagnostic method. This study was performed to explore the relationship between the qPCR findings, clinical symptoms, and inflammatory markers in children with MPP. Four hundred children with MPP have been enrolled in this retrospective analysis. All clinical and analytical information, including mycoplasma pneumoniae (MP) PCR results, has been collected. Based on the PCR results, the patients were divided into groups with load values (copy number) $<10^{5}$ (54 cases), $\geq 10^{5}$ and $<10^{6}$ (71 cases), $\geq 10^{6}$ and $<10^{7}(112$ cases), $\geq 10^{7}$ and $\leq 10^{8}$ (114 cases), and $>10^{8}$ (49 cases). The clinical features (including symptoms and signs) and inflammatory indicators were compared among the groups. The incidence of high fever (above $39^{\circ} \mathrm{C}$ ), thermal peak during the entire hospitalization period, fever duration, days of hospitalization, and plasma lactate dehydrogenase (LDH) levels were statistically correlated with the MP PCR load value in children with MPP. The analysis of relevance degree showed the correlative order as a thermal peak of hospitalization $>$ duration of fever $>$ period of hospitalization $>\mathrm{LDH}$ value $>\mathrm{C}$-reactive protein value. The host immune response was significantly greater in the complication group than in the non-complication group.
\end{abstract}

Key words: mycoplasma pneumonia; quantitative polymerase chain reaction; bacterial load

Mycoplasma pneumoniae (MP) is a common cause of community-acquired pneumonia and upper respiratory tract infections, particularly in children and adolescents, and accounts for approximately 10\%$30 \%$ of all community-acquired pneumonia ${ }^{[1]}$. MP pneumonia (MPP) is considered a self-limited disease; however, it may cause various pulmonary symptoms, such as fever, dry cough, dyspnea, wheezing, etc. Extrapulmonary complications induced by MPP can lead to severe life-threatening disorders ${ }^{[2-4]}$. The onset of MPP is usually sudden and unpredictable with non-specific symptoms, such as headache, malaise, and emesis ${ }^{[5]}$.

Chen ZHANG, E-mail: 406091854@qq.com; Qiao ZHANG, E-mail: qiaoz@hospital.cqmu.edu.cn

${ }^{\dagger}$ The authors contributed equally to this study.

\#Corresponding author, E-mail: 400942@hospital.cqmu.edu. cn

${ }^{*}$ This study was supported by the Chongqing Science and Health Joint Medical Research Project (No. 8187011078)
The radiological features of the extra-pulmonary complications of MPP are often highly variable in patients. Pleural effusion has been shown to occur in $20 \%$ of patients with MPP by standard, posterioranterior, and lateral chest radiographs ${ }^{[6]}$. In the early stages, MPP can be easily misdiagnosed clinically due to lack of symptoms, specific clinical features, and consistency in radiological manifestations ${ }^{[3,7]}$.

Since MP does not have a cellular wall, it is not sensitive to most antibiotics. However, erythromycin, clarithromycin, azithromycin, and tetracyclines exhibit effective in vitro bactericidal activity against MP. The most effective option for shortening the progress of mycoplasma diseases are macrolides ${ }^{[8]}$. Indeed, clinical studies have shown that an early administration of macrolides can reduce the duration of MPP symptoms ${ }^{[9]}$. Corticosteroids can be used for treating MPP cases with hyperactive immune response ${ }^{[11-13]}$ as they inhibit the inflammatory response ${ }^{[10]}$. 
There are three methods used to detect MP worldwide: sputum culture, quantitative polymerase chain reaction (qPCR), and serological tests. Among them, $\mathrm{qPCR}$ is more convenient, sensitive and provides fast results than the other two methods and may even expose the subtype of $\mathrm{MP}^{[14]}$; thus, it is commonly used in clinical practices.

With the growing infection of MP, it is necessary to investigate the relationship of the bacterial load, clinical severity, and inflammatory reaction in children with MPP to understand the clinical characteristics of the disease and strengthen the diagnosis, therapy, and prognosis. Since qPCR has high precision and quantifies bacterial loads, MPP is diagnosed using realtime PCR. In this analysis, children with MP-positive results in $\mathrm{qPCR}$ were included in a retrospective review of the relationships between the quantitative value obtained from qPCR, clinical manifestations, and inflammatory markers to predict the severity of clinical manifestations and the inflammation reaction on the qPCR results.

\section{PATIENTS AND METHODS}

\subsection{MPP Cases}

The medical records of 570 children with MPP admitted to the Children's Hospital of Chongqing Medical University in China were collected between August 2008 and December 2016. Based on the Clinical Diagnostic Criteria of MPP in Pediatrics (9th edition) published by People's Health Publishing House ${ }^{[15]}$, patients with respiratory symptoms (such as cough, fever, tachypnea, cyanosis, wheezing), abnormal breath sounds during auscultation examination, and chest X-ray abnormalities were included in this study. The diagnosis of MP infection was based on positive MP qPCR results (value $>4 \times 10^{2}$ ) from the patient's respiratory tract sputum. Patients who were co-infected with other pathogens, and reported positive results from bacterial cultures of respiratory secretions or blood samples and fluorescent assays for respiratory virus detection, were excluded from this study. Patients with underlying diseases, such as asthma, recurrent respiratory tract infection, chronic cardiac and pulmonary diseases, rheumatic diseases, and immunodeficiency, were excluded. Patients with missing or ambiguous information were also excluded $^{[16]}$. Finally, 400 patients remained. Use of the database for this retrospective study was approved and permitted by the Ethics Committee of the Children's Hospital of Chongqing Medical University.

\subsection{Data Collection and Laboratory Examinations}

All information recorded by physicians and nurses during hospitalization, including demographic and clinical information, laboratory examinations, and chest radiographic and sonographic results were reviewed and extracted. All the above data were reviewed, identified, and anonymously analyzed by the author.

\section{3 qPCR for Investigation of MP and Other Pathogens}

After admission, sputum specimens from the patients were immediately collected from the respiratory tract using a sputum suction tube for MP analysis, bacterial culture, and virus detection. DNA extracting kit (Sansure Biotech, China) was used for extracting MP DNA in the sputum. The copy number for the MP DNA was detected using real-time PCR following the protocol provided by Real-time PCR Fluorescence Detection Systems (Thermo Fisher Scientific, USA) in an ABI 7500 quantitative PCR analyzer (Applied Biosystems, USA). The upstream MP primer was found as 5'-AGATCAACTTCTGTCATCCAGCAA-3', and the downstream primer was $5^{\prime}$-TTCTGCACATCATA ATTAGGAGTATCAAT-3'.

Using direct immunofluorescence assays (DHI, 1055 East State St., Suite 100, USA), the sputum specimens were tested for seven viruses. Briefly, the patient's throat was swiped with a swab, which was placed in a $2 \mathrm{~mL}$ PBS-containing $(15 \mathrm{~mL})$ tube. The tube was vortexed for 30 , and then the swab was removed. $200 \mu \mathrm{L}$ of the sample was transferred into each of seven wells in a 24 -well plate. Subsequently, $25 \mu \mathrm{L}$ of the corresponding fluorescent antibody regent (total of seven regents) was added to each well separately, followed by $30 \mathrm{~min}$ incubation. After washing with washing buffer, the cells in the sample mixture were centrifuged at $700 \mathrm{~g}$ for $2 \mathrm{~min}$, smeared on a glass slide, and covered with a cover glass after adding a drop of sealing solution. The stained (positive) cells were checked under a fluorescence microscope and the ratio of the number of positive cells to the total number of cells was calculated .

\subsection{Hematological and Biochemical Analysis}

Peripheral blood samples from patients were obtained to determine the complete blood count. The C-reactive protein (CRP) level was measured using a CRP rapid quantitative kit (Shanghai Op Biological, China). The Enzyme Kinetic Kit (ROCHE Diagnosis, Switzerland) was used to examine lactate dehydrogenase (LDH).

\subsection{Treatment}

The majority of patients were treated with macrolides (azithromycin or erythromycin). Simultaneously, some severe cases were additionally treated with a broad-spectrum antibiotic (ceftriaxone or amoxicillin) and/or corticosteroid based on the condition.

\subsection{Classification}

The patients were classified into five groups based on the copy number of MP genes determined using qPCR: low copy group $\left(<10^{5}\right.$ copies, 54 cases); low-medium copy group $\left(\geq 10^{5}-<10^{6}\right.$ copies 71 cases $)$; medium copy group $\left(\geq 10^{6}-<10^{7}\right.$ copies, 112 cases); 
medium-high copy group $\left(\geq 10^{7}-\leq 10^{8}\right.$ copies, 114 cases); high copy group ( $>10^{8}$ copies, 49 cases). The patients were classified into male/female, wheezing, fever $\left(\geq 37.5^{\circ} \mathrm{C}\right)$, high fever $\left(\geq 39.1^{\circ} \mathrm{C}\right)$, tachypnea, inspiratory tri-concave sign, cyanosis, pleural effusion, and atelectasis groups based on the clinical symptoms and signs. The linear relationships between the qPCR quantitative value and other variables, including patient's age, weight, hospitalization period, thermal peak during entire period of hospitalization, fever duration, respiratory frequency, leukocyte count, CRP, LDH levels, alanine aminotransferase (ALT), aspartate aminotransferase (AST), albumin, and urea nitrogen were also analyzed. The clinical results were divided into five categories: atelectasis, pleural effusion, high fever, hormone use, and hospitalization days for more than 7 days. The differences of inflammatory indicators (CRP and LDH) values among five categories were compared.

\subsection{Clinical Sign Definitions}

Cyanosis was determined based on the lips and/or nails. Inspiratory three concave sign was characterized as apparent depression in the suprasternal fossa, supraclavicular fossa, and rib space during inspiration. During hospitalization, pleural effusion and atelectasis were assessed using standard, posterior-anterior, and lateral chest X-rays and ultrasound. Tachypnea was identified among children with different ages: aged $<2$ months with a respiratory rate $(\mathrm{RF}) \geq 60$ times per minute; $2-12$ months of age with $\mathrm{RF} \geq 50$ times per minute; $1-5$ years of age with $R F \geq 40$ times per minute; $>5$ years of age with $\mathrm{RF} \geq 30$ times per minute. A febrile illness was defined when the body temperature exceeded $37.5^{\circ} \mathrm{C}^{[17]}$.

\subsection{Statistical Analysis}

The data were analyzed using the software SPSS version 24.0. Correlations were determined using Spearman correlation analysis. In order to evaluate the degree of correlation, the grey correlation model was used, and higher correlation coefficients between 0 and 1 suggested a stronger correlation. The relational order represents the degree of correlation, as calculated in the grey correlation mode. Higher degrees of correlation between a variable and strain indicate a larger contribution to the strain from the variable. The counting data were tested with the Chi-square test. Mann Whitney $U$ test was used to compare the variation between the two groups. $P<0.05$ was found to be significantly different.

\section{RESULTS}

\subsection{Comparison of Non-quantitative Data to qPCR Values}

Table 1 presents the demographic information for patients of whom $54.8 \%(n=219)$ were male (male to female ratio was 1:0.83). For antibiotic treatment, macrolides are recommended as first-line drugs for children with MPP, and $28.7 \%$ of patients $(n=115)$ used macrolides prior to admission. As macrolides are commonly used in the treatment of MP, they have also been continued after admission. The four most common symptoms were fever $(n=372,93 \%)$, tachypnea $(n=145,36.2 \%)$, cyanosis $(n=139,34.7 \%)$, and wheezing $(n=45,11.2 \%)$. The majority $(72.3 \%)$ of patients had a low-middle fever $\left(\leq 39^{\circ} \mathrm{C}\right.$ and $\left.\geq 37.5^{\circ} \mathrm{C}\right)$. Chest radiograph showed $16.2 \%(n=65)$ and $19.7 \%$ $(n=79)$ of cases progressed to atelectasis and pleural

Table 1 Comparison of symptoms and signs among different PCR groups

\begin{tabular}{|c|c|c|c|c|c|c|c|c|c|}
\hline \multirow{2}{*}{ Variables } & & \multirow{2}{*}{$\begin{array}{l}\text { Cases } \\
n(\%)\end{array}$} & \multicolumn{5}{|c|}{ PCR values (copies) } & \multirow{2}{*}{$\chi^{2}$} & \multirow{2}{*}{$P$} \\
\hline & & & $<10^{5}$ & $\geq 10^{5}$ and $<10^{6}$ & $\geq 10^{6}$ and $<10^{7}$ & $\geq 10^{7}$ and $\leq 10^{8}$ & $>10^{8}$ & & \\
\hline \multirow[t]{2}{*}{ Gender } & Female & $181(45.2 \%)$ & $23(42.6 \%)$ & $29(40.8 \%)$ & $43(38.4 \%)$ & $63(55.3 \%)$ & $23(46.9 \%)$ & 7.506 & 0.111 \\
\hline & Male & $219(54.8 \%)$ & $31(57.4 \%)$ & $42(59.2 \%)$ & $69(61.6 \%)$ & $51(44.7 \%)$ & $26(53.1 \%)$ & & \\
\hline \multirow{2}{*}{$\begin{array}{l}\text { Inspiratory three } \\
\text { concave sign }\end{array}$} & No & $379(94.8 \%)$ & $52(96.3 \%)$ & $68(95.8 \%)$ & $107(95.5 \%)$ & $104(91.2 \%)$ & $48(98.0 \%)$ & 4.406 & 0.35 \\
\hline & Yes & $21(5.2 \%)$ & $2(3.7 \%)$ & $3(4.2 \%)$ & $5(4.5 \%)$ & $10(8.8 \%)$ & $1(2.0 \%)$ & & \\
\hline \multirow[t]{2}{*}{ Cyanosis } & No & $261(65.3 \%)$ & $33(61.1 \%)$ & $38(53$ & $84(75$. & $75(6$ & $31(63.3 \%)$ & 9.511 & 0.05 \\
\hline & Yes & $139(34.7 \%)$ & $21(38.9$ & & 28( & & $18(36$ & & \\
\hline \multirow[t]{2}{*}{ Pleural effusion } & No & $321(80.3 \%)$ & $41\left(75.9^{\circ}\right.$ & $57(8$ & $94(83.9$ & $90(7$ & $39(79.6 \%)$ & 1.729 & 0.78 \\
\hline & Yes & $79(19.7 \%)$ & $13(24.1$ & & & & $10(20$. & & \\
\hline \multirow[t]{2}{*}{ Atelectasis } & No & $335(83.8 \%)$ & $43(79.6 \%)$ & $62(87$. & $90(8$ & 100( & $40(81.6 \%)$ & 3.769 & 0.43 \\
\hline & Yes & $65(16.2 \%)$ & $11(20.4 \%)$ & & & & & & \\
\hline \multirow{2}{*}{$\begin{array}{l}\text { Treatment of antibiotics } \\
\text { outside hospital }\end{array}$} & No & $285(71.3 \%)$ & $37(68.5 \%)$ & $49(69.0 \%)$ & $77(c$ & 83 & $39(79.6 \%)$ & 2.511 & 0.64 \\
\hline & Yes & $115(28.7 \%)$ & $17(31.5 \%)$ & & & & & & \\
\hline \multirow[t]{2}{*}{ Wheezing } & No & $355(88.8 \%)$ & $48(88.9 \%)$ & $66(93.0 \%)$ & $98(87.5 \%)$ & $101(88.6 \%)$ & $42(85.7 \%)$ & 1.890 & 0.75 \\
\hline & Yes & $45(11.2 \%)$ & & & & $13(11.4 \%)$ & & & \\
\hline \multirow[t]{2}{*}{ Fever $\left(\geq 37.5^{\circ} \mathrm{C}\right)$} & No & $28(7 \%)$ & $5(9.3 \%)$ & $5(7.0 \%)$ & $10(8.9 \%)$ & $7(6.1 \%)$ & $1(2.0 \%)$ & 3.081 & 0.54 \\
\hline & Yes & & & & $102(91.1 \%)$ & & & & \\
\hline \multirow[t]{2}{*}{ High fever $\left(\geq 39.1^{\circ} \mathrm{C}\right)$} & No & $289(72.3 \%)$ & $46(85.2 \%)$ & $48(67.6 \%)$ & $84(75.0 \%)$ & $86(75.4 \%)$ & $25(51.0 \%)$ & 17.286 & 0.00 \\
\hline & Yes & $111(27.7 \%)$ & $8(14.8 \%)$ & $23(32.4 \%)$ & $28(25.0 \%)$ & $28(24.6 \%)$ & $24(49.0 \%)$ & & \\
\hline \multirow[t]{2}{*}{ Tachypnea } & No & $255(63.8 \%)$ & $37(68.5 \%)$ & $50(70.4 \%)$ & $68(60.7 \%)$ & $69(60.5 \%)$ & $31(63.3 \%)$ & 2.863 & 0.85 \\
\hline & Yes & $145(36.2 \%)$ & $17(31.5 \%)$ & $21(29.6 \%)$ & $44(39.3 \%)$ & $45(39.5 \%)$ & $18(36.7 \%)$ & & \\
\hline
\end{tabular}


effusion, respectively. The Chi-square test was used to identify discrepancies in observed items among the groups and showed that there were no significant differences in the gender distribution, three concave sign, cyanosis, tachypnea, wheezing, X-ray atelectasis, pleural effusion on ultrasound, and out-patient with antibiotic treatment among the different groups of patients with MPP $(P \geq 0.05)$. Only high fever was found to have significant differences $(P<0.01)$, suggesting that high fever $\left(\geq 39.1^{\circ} \mathrm{C}\right)$ was positively associated with a high copy of MPP loading (table 1). This finding is consistent with the subsequent linear relationship analysis that a higher value of qPCR resulted in a higher thermal peak during the entire duration of hospitalization.

\subsection{Comparison of the Quantitative Data to qPCR Values}

Variance analysis was used to evaluate the distribution of continuous variables among the PCR groups. The days of hospitalization, thermal peak during the entire period of hospitalization, urea nitrogen, and LDH among groups with different PCR copy numbers were statistically different $(P<0.05)$ (table 2). However, there were no significant differences in age, weight, duration of fever, leukocyte number, neutrophil ratio, lymphocyte proportion, CRP, AST, ALT, albumin, and creatinine among the groups $(P<0.05)$ (table 2). In order to examine the correlation among variables of the quantitative indexes and MP PCR loadings, Spearman's rank correlation coefficient analysis was used, as shown in table 3 . The results indicated that the PCR value was only associated with the days of hospitalization, thermal peak during the entire period of hospitalization, duration of fever, lymphocyte ratio, CRP, and LDH $(P<0.05)$. The higher the loading of qPCR, the longer the hospitalization period, the higher the thermal peak, the CRP, and the LDH levels, the longer the duration of fever, and the lower the lymphocyte ratio. The grey correlation model was used to analyze the degree of correlation between the MP PCR loading and the correlated indexes above (table 3). The correlative order from strong to weak was as follows: thermal peak during hospitalization $>$ lymphocyte ratio $>$ fever duration $>$ days of hospitalization > LDH level > CRP level. The period of hospitalization, thermal peak during the entire period of hospitalization, and LDH levels were positively correlated with the PCR values, as the data were presented in tables 2 and 3. While CRP and fever duration were positively correlated with PCR values; however, there was no significant difference between the PCR groups, suggesting that the correlation was weak.

\subsection{Analysis of Host Immune Response and Clinical Severity}

The Mann-Whitney $U$ Test was used to compare

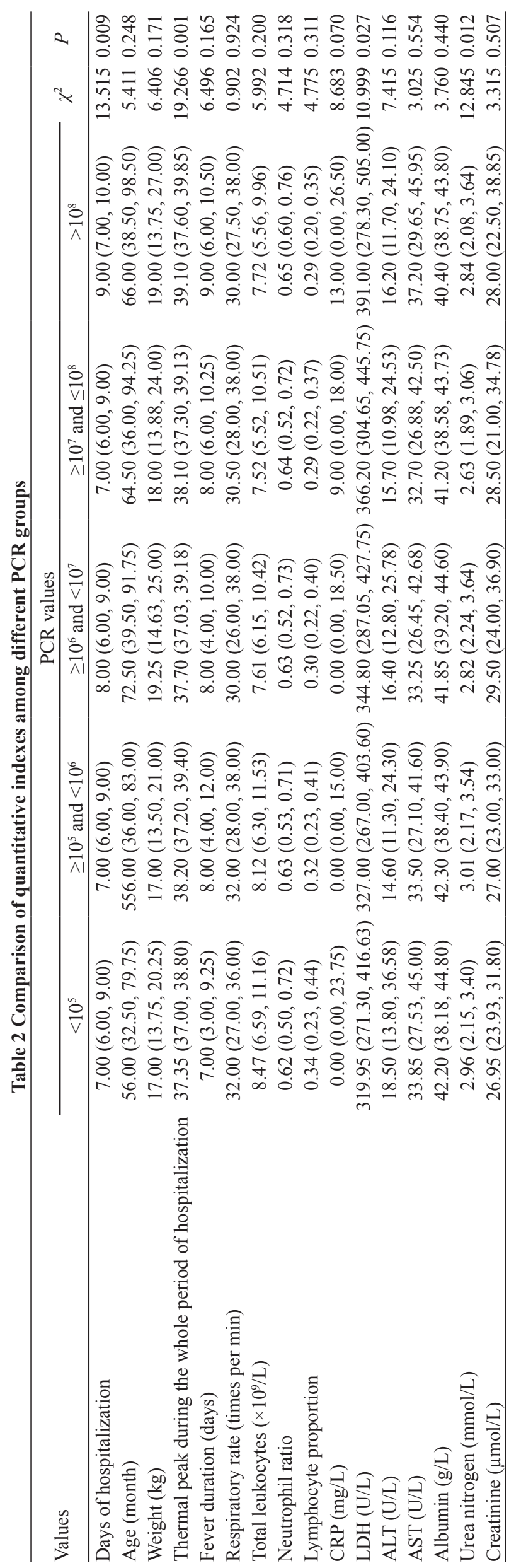


Table 3 Analysis of correlation degree between each correlative variable and PCR

\begin{tabular}{|c|c|c|c|c|c|c|c|}
\hline Variables & & $\begin{array}{l}\text { Thermal peak during } \\
\text { the whole period of } \\
\text { hospitalization }\end{array}$ & $\begin{array}{l}\text { Lymphocyte } \\
\text { ratio }\end{array}$ & $\begin{array}{c}\text { Fever } \\
\text { duration }\end{array}$ & $\begin{array}{c}\text { Days of } \\
\text { hospitalization }\end{array}$ & $\begin{array}{l}\mathrm{LDH} \\
(\mathrm{U} / \mathrm{L})\end{array}$ & $\begin{array}{c}\text { CRP } \\
(\mathrm{mg} / \mathrm{L})\end{array}$ \\
\hline \multirow[t]{2}{*}{ Association with PCR } & Correlation coefficient & $0.179^{* *}$ & $-0.107^{*}$ & $0.110^{*}$ & $0.148^{* *}$ & $0.160^{* *}$ & $0.105^{*}$ \\
\hline & $P$ value & 0.000 & 0.000 & 0.028 & 0.003 & 0.001 & 0.035 \\
\hline \multirow[t]{2}{*}{ Correlation } & Order & 1 & 2 & 3 & 4 & 5 & 6 \\
\hline & Degree & 0.640 & 0.639 & 0.620 & 0.617 & 0.576 & 0.559 \\
\hline
\end{tabular}

The correlation coefficient is the degree of correlation between each independent variable and the strain.

${ }^{* *}: P<0.01$ (two-sided test); ${ }^{*}: P<0.05$ (two-sided test).

Order refers to the degree of the correlation of the factor and the value of PCR from strong to weak.

A thermal peak during the whole period of hospitalization refers to the monitoring of body temperature every day during hospitalization.

The highest temperature measured is the peak fever during hospitalization.

Table 4 Analysis of inflammatory indicators and clinical severity

\begin{tabular}{|c|c|c|c|c|c|c|c|c|}
\hline \multirow{2}{*}{ Variables } & & \multirow{2}{*}{ Cases } & \multicolumn{3}{|c|}{ CRP } & \multicolumn{3}{|c|}{$\mathrm{LDH}$} \\
\hline & & & Mean rank & $Z$ & $P$ & Mean rank & $Z$ & $P$ \\
\hline \multirow[t]{2}{*}{ Pleural effusion } & Yes & 79 & 274.75 & -6.883 & $<0.001$ & 262.53 & -5.323 & $<0.001$ \\
\hline & No & 321 & 182.23 & & & 185.24 & & \\
\hline \multirow[t]{2}{*}{ Atelectasis } & Yes & 65 & 224.18 & -1.949 & 0.051 & 231.68 & -2.376 & 0.017 \\
\hline & No & 335 & 195.91 & & & 194.45 & & \\
\hline \multirow[t]{2}{*}{ High fever $\left(\geq 39.1^{\circ} \mathrm{C}\right)$} & Yes & 111 & 256.59 & -6.494 & $<0.001$ & 264.95 & -6.910 & $<0.001$ \\
\hline & No & 289 & 178.96 & & & 175.75 & & \\
\hline \multirow[t]{2}{*}{ Glucocorticoid usage } & Yes & 34 & 230.84 & -1.728 & 0.084 & 278.54 & -4.115 & $<0.001$ \\
\hline & No & 366 & 197.68 & & & 193.25 & & \\
\hline \multirow[t]{2}{*}{ Days of hospitalization $>7$ days } & Yes & 211 & 214.85 & -2.832 & 0.005 & 220.09 & -3.581 & $<0.001$ \\
\hline & No & 189 & 184.48 & & & 178.63 & & \\
\hline
\end{tabular}

CRP and LDH values between two groups. The data presented in table 4 suggest that there was a statistically significant difference $(P<0.05)$ in CRP between pleural effusion group and non-pleural effusion group, between high fever group and non-high fever group, and between more than 7 days of hospitalization group and no more than 7 days of hospitalization group $(P<0.05)$. However, no significant difference was observed between atelectasis groups and glucocorticoid usage groups $(P>0.05)$, whereas the variation in LDH values among all variable groups was statistically significant $(P<0.05)$. Furthermore, the mean rank of CRP and LDH in the positive symptom group was higher than that in the negative symptom group. The cases with complications, such as atelectasis, pleural effusion, as well as high fever, glucocorticosteroids used to regulate the immune response, and more than 7 days of hospitalization, are considered as having severe lung damage.

\section{DISCUSSION}

MP, formerly known as pleuropneumonia-like microorganism (PPLO), is a cell-wall-free pathogen between virus and bacteria that can move through bacterial filters $\left.{ }^{[9,} 18\right]$. Invading pathogens and the overwhelming immune response are both critical factors in the pathogenesis of MPP. When MP enters human respiratory tract, the surface lipoproteins interact with respiratory epithelial cells to activate the host immune system by stimulating the synthesis of intracellular adhesion molecule receptors via Toll-like receptors or TLR-4. The activated macrophages secrete pro-inflammatory cytokines and synthesize respective proteins. As a result, the stronger the cell-mediated immune response and stimulation of cytokine, the more severe the clinical disease and lung injury. Thus, both bacterial load and host immune system interactions are suggested to result in various pathogenic patterns, chest imaging, and inflammatory responses ${ }^{[8,19-24]}$. Hence, we used qPCR for MP identification, and based on qPCR results, we observed correlations between PCR loading and the clinical manifestations, signs, and inflammatory indicators.

We found that only high fever $\left(>39.1^{\circ} \mathrm{C}\right)$ showed a significant difference after comparing the clinical manifestations among the groups, suggesting that higher bacterial MP loads resulted in a high fever. There was no significant difference between PCR loading and fever, and other clinical symptoms, such as gender, inspiratory three concave sign, wheezing, tachypnea, and cyanosis among the groups. Fever is a defensive response by our body. Most patients (93\%) infected with MP exhibited fever regardless of the copy load of bacteria. Infections of various pathogens cause different types of fever. MP infection typically results in a low fever based on the present data. However, high fever can occur in response to the load of the bacterial 
infection that triggers an immune response and other reasons. Persistent fever can damage cells in the body, especially neural cells in the brain. As a result, high fever can be used as an indicator to predict the severity of clinical manifestation. Our data showed that the high fever ratio in the high copy loading group was higher than that in the low copy loading group, suggesting that MPP patients with high copy loads should be alerted to lung injury caused by high fever.

Furthermore, the correlation between MP copy load and high fever in MPP patients was confirmed by linear relationship analysis. During the entire period of hospitalization and longer fever duration, higher PCR values were correlated with higher thermal peaks. The prolonged high fever would injure the body, thereby requiring longer recovery time, and consequently, longer hospitalization. However, it is possible to predict the type of fever (the temperature and duration) and the days of hospitalization for patients with MPP based on the quantitative value obtained from MP PCR. Thus, the MP PCR load may serve as a guideline to accurately predict the prognosis of MPP, take symptomatic treatment on time, and help doctors explain the condition of patients. Interestingly, these results are consistent with those obtained by Nilsson et al. Their data suggested that the MP bacterial count in oral and oropharyngeal secretions, determined using PCR from children with MPP, was significantly higher in 24 hospitalized children than in 21 out-patient children with MPP. However, no difference in MP genotype distribution was observed between the outpatient group and in-patient group. Therefore, they concluded that the clinical severity of MP infection was not related to the MP genotype but the MP bacterial count $^{[25]}$.

Our results also showed no significant difference between MP loading and other clinical characteristics, including gender, inspiratory three concave sign, wheezing, tachypnea, and cyanosis among the different PCR load groups. Higher PCR values are also expected to suggest invasive bacteria and more severe complications of pneumonia. However, this has not always been the case in our research. Some children with low copy numbers in MP PCR had severe clinical symptoms, such as cyanosis, tachypnea, and dyspnea, and even required intubation. Besides, we found that pulmonary complications, including atelectasis and pleural effusion, did not show significant differences among the PCR groups. Hence, physicians need to be careful of all patients who have positive MP qPCR results and should closely monitor the vital signs of children.

MP has been reported to cause alterations in a variety of serum immune indicators. Due to limited parental authorization, blood samples were collected from few patients with MPP for routine blood tests and biochemical analysis (leukocyte counting, lymphocyte ratio, neutrophil ratio, albumin, $\mathrm{LDH}, \mathrm{CRP}$, liver function test, kidney function test, etc.). We found that CRP and LDH were positively correlated with the MP loading, as presented in table 2. Higher PCR values for MP are associated with higher CRP and LDH values. The number of lymphocytes is negatively correlated with the MP PCR value, which may be related to immunosuppression following MP infection. However, people with a reduced number of lymphocytes might be more susceptible to MP after getting the infection and more likely to progress to pneumonia ${ }^{[17,26]}$. If the results find that other indexes, such as leukocyte count, albumin, liver function test results, and kidney function test remain within the normal range, MPP is considered a mild, self-limiting disease, and rarely damages other systems. CRP and LDH are acute-phase proteins that are synthesized by the organism when stimulated by inflammation. Bacterial infection or tissue damage can result in a significant increase in CRP and LDH; thus, sensitive measures of inflammatory reaction and tissue damage are considered.

The present study showed that the more severe the lung injury, the greater secretion of associated inflammatory indicators (LDH and CRP). The CRP and LDH levels in the children with pleural effusion and atelectasis were significantly higher than those in the non-complication group, suggesting that severe MP infection with high fever, long hospital stay, and the use of glucocorticoids to control the disease have a stronger immune response. As a result, a higher inflammatory response of the body results in a stronger cell-mediated immune response and activation of cytokine and a more severe pulmonary injury ${ }^{[19-21]}$. This information can help to detect refractory MP at an early clinical stage.

In conclusion, the PCR loading of MP is associated with clinical severity and inflammation indicators. The high bacterial load group suggests that high fever, high thermal peak, long duration of fever, and elevated levels of CRP and LDH result in low lymphocyte ratios and serious lung damages. Therefore, it is important for patients to extend the hospital stay and improve the treatment of immunity and anti-infection.

There is a lack of research on the correlation between the PCR value of MP in sputum and clinical severity, inflammatory factors worldwide, and the sample size of most studies is less than 100. No accurate and reliable conclusion has been drawn so far. Hence, we carried out this study and collected about 400 samples after excluding the unqualified cases. Furthermore, the data collected in this study can show the law of the natural lesion process, and suggest the direction of future research. The results obtained from the present analysis will play a significant role in guiding medical and public health workers as well 
as researchers. However, there are several limitations to this study. Firstly, it is a retrospective study, which appears to have a selection bias. Secondly, due to shortcomings in the existing detection technology, the presence of other pathogens could not be detected. Thirdly, the distribution of the number of patients among the five groups could not be balanced, which could have influenced the statistical analysis. Fourthly, the number of cases in each group was not very high; thus, research on multicenter, large sample size is required to be carried out in future studies.

\section{Conflict of Interest Statement}

The authors declare that there is no conflict of interest with any financial organization or corporation or individual that can inappropriately influence this work.

\section{REFERENCES}

1 Yoon IA, Hong KB, Lee HJ, et al. Radiologic findings as a determinant and no effect of macrolide resistance on clinical course of Mycoplasma pneumoniae pneumonia. BMC Infect Dis, 2017,7(1):402

2 Kim CK, Kim SW, Kim JS, et al. Bronchiolitis obliterans in the 1990s in Korea and the United States. Chest, 2001,120(4):1101-1106

3 Wang RS, Wang SY, Hsieh KS, et al. Necrotizing pneumonitis caused by Mycoplasma pneumoniae in pediatric patients: report of five cases and review of literature. Pediatr Infect Dis J, 2004,23(6):564-567

4 Hawkins S, Rausch CM, McCanta AC. Constrictive pericarditis secondary to infection with Mycoplasma pneumoniae. Curr Opin Pediatr, 2011,23(1):126-129

5 Montagnani F, Rossetti B, Vannoni A, et al. Laboratory diagnosis of Mycoplasma pneumoniae infections: Data analysis from clinical practice. New Microbiol, 2018, 41(2):203-207

6 Socan M, Ravnik I, Bencina D, et al. Neurological symptoms in patients whose cerebrospinal fluid is culture- and/or polymerase chain reaction-positive for Mycoplasma pneumoniae. Clin Infect Dis, 2001,32(2): 31-35

7 Chen Y, Tian WM, Chen Q, et al. Clinical features and treatment of macrolide-resistant Mycoplasma pneumoniae pneumonia in children. Zhongguo Dang Dai Er Ke Za Zhi (Chinese), 2018,20(8):629-634

8 Radisic M, Torn A, Gutierrez P, et al. Severe acute lung injury caused by Mycoplasma pneumoniae: potential role for steroid pulses in treatment. Clin Infect Dis, 2000,31(6):1507-1511

9 Purighalla R, Shapiro R, McCauley J, et al. BK virus infection in a kidney allograft diagnosed by needle biopsy. Am J Kidney Dis, 1995,26(4):671-673

10 Pereyre S, Goret J, Bebear C. Mycoplasma pneumoniae: Current Knowledge on Macrolide Resistance and Treatment. Front Microbiol, 2016,7(6):974

11 Azumagawa K, Kambara Y, Murata T, et al. Four cases of arthritis associated with Mycoplasma pneumoniae infection. Pediatr Int, 2008,50(1):511-513

12 Khan FY, M Ay. Mycoplasma pneumoniae associated with severe autoimmune hemolytic anemia: case report and literature review. Braz J Infect Dis, 2009,13(1):7779

13 Takiguchi Y, Shikama N, Aotsuka N, et al. Fulminant Mycoplasma pneumoniae pneumonia. Intern Med, 2001,40(4):345-348

14 Li X, Chen H, You L, et al. Evaluation of the commercial rapid Mycoplasma pneumoniae medium method using the medium PCR and serum antibody test. Clin Lab, 2011,57(6):351-355

15 Zhao DY, Chen HZ, Zheng YJ. The diagnosis of Mycoplasma pneumoniae infections. Zhonghua Er Ke Za Zhi (Chinese), 2016,54(2):98-100

16 Sorensen CM, Schonning K, Rosenfeldt V. Clinical characteristics of children with Mycoplasma pneumoniae infection hospitalized during the Danish 2010-2012 epidemic. Dan Med J, 2013,60(5):A4632

17 Youn YS, Lee KY, Hwang JY, et al. Difference of clinical features in childhood Mycoplasma pneumoniae pneumonia. BMC Pediatr, 2010,10:48

18 Lee E, Cho HJ, Hong SJ. Prevalence and clinical manifestations of macrolide resistant Mycoplasma pneumoniae pneumonia in Korean children. Korean J Pediatr, 2017,60(5):151-157

19 Parrott GL, Kinjo T, Fujita J. A Compendium for Mycoplasma pneumoniae. Front Microbiol, 2016,7(3): 513

20 Waites KB, Talkington DF. Mycoplasma pneumoniae and its role as a human pathogen. Clin Microbiol Rev 2004,17(4):697-728

21 Waites KB, Balish MF, Atkinson TP. New insights into the pathogenesis and detection of Mycoplasma pneumoniae infections. Future Microbiol, 2008,3(6):635-648

22 Meyer Sauteur PM, Unger WW, Nadal D, et al. Infection with and Carriage of Mycoplasma pneumoniae in Children. Front Microbiol, 2016,3(7):329

23 Yamazaki T, Kenri T. Epidemiology of Mycoplasma pneumoniae Infections in Japan and Therapeutic Strategies for Macrolide-Resistant M. pneumoniae. Front Microbiol, 2016,7:693

24 Wu PS, Chang LY, Lin HC, et al. Epidemiology and clinical manifestations of children with macrolideresistant Mycoplasma pneumoniae pneumonia in Taiwan. Pediatr Pulmonol, 2013,48(9):904-911

25 Nilsson AC, Bjorkman P, Welinder-Olsson C, et al. Clinical severity of Mycoplasma pneumoniae (MP) infection is associated with bacterial load in oropharyngeal secretions but not with MP genotype. BMC Infect Dis, 2010,25(10):39

26 Stelmach I, Podsiadlowicz-Borzecka M, Grzelewski T, et al. Humoral and cellular immunity in children with Mycoplasma pneumoniae infection: a 1-year prospective study. Clin Diagn Lab Immunol, 2005,12(10):12461250

(Received Aug. 10, 2020; accepted Sep. 8, 2020) 